\section{D) Check for updates}

Cite this: Nanoscale, 2020, 12, 20220

Received 24th August 2020, Accepted 23rd September 2020 DOI: $10.1039 / \mathrm{d} 0 \mathrm{nr} 06139 \mathrm{e}$

rsc.li/nanoscale

\title{
The role of carbon dots - derived underlayer in hematite photoanodes $\uparrow$
}

\author{
Qian Guo, ${ }^{a}$ Hui Luo, ${ }^{b}$ Jifang Zhang, ${ }^{c}$ Qiushi Ruan, (D) d Arun Prakash Periasamy, ${ }^{a}$ \\ Yuanxing Fang, (D) ${ }^{e}$ Zailai Xie, (D) ${ }^{\text {e }}$ Xuanhua Li, (D) ${ }^{f}$ Xinchen Wang, (D) e \\ Junwang Tang, (D) d Joe Briscoe, (D) ${ }^{a}$ Magdalena Titirici (iD ${ }^{\mathrm{b}}$ and \\ Ana Belen Jorge (iD) *a
}

\begin{abstract}
Hematite is a promising candidate as photoanode for solar-driven water splitting, with a theoretically predicted maximum solar-to-hydrogen conversion efficiency of $\sim 16 \%$. However, the interfacial charge transfer and recombination greatly limits its activity for photoelectrochemical water splitting. Carbon dots exhibit great potential in photoelectrochemical water splitting for solar to hydrogen conversion as photosensitisers and co-catalysts. Here we developed a novel carbon underlayer from low-cost and environmental-friendly carbon dots through a facile hydrothermal process, introduced between the fluorinedoped tin oxide conducting substrate and hematite photoanodes. This led to a remarkable enhancement in the photocurrent density. Owing to the triple functional role of carbon dots underlayer in improving the interfacial properties of FTO/hematite and providing carbon source for the overlayer as well as the change in the iron oxidation state, the bulk and interfacial charge transfer dynamics of hematite are significantly enhanced, and consequently led to a remarkable enhancement in the photocurrent density. The results revealed a substantial improvement in the charge transfer rate, yielding a charge transfer efficiency of up to $80 \%$ at $1.25 \mathrm{~V}$ vs. RHE. In addition, a significant enhancement in the lifetime of photogenerated electrons and an increased carrier density were observed for the hematite photoanodes modified with a carbon underlayer, confirming that the use of sustainable carbon nanomaterials is an effective strategy to boost the photoelectrochemical performance of semiconductors for energy conversion.
\end{abstract}

\section{Introduction}

Due to their low cost and non-toxicity, carbon materials have been widely developed for a broad range of application across multiple fields, from energy and optoelectronic devices to sensors. ${ }^{1-6}$ Recently, one particular field where carbons find new potential is in photoelectrochemical (PEC) water splitting,

\footnotetext{
${ }^{a}$ School of Engineering and Materials Science, Queen Mary University of London, E1 4NS London, UK. E-mail: a.sobrido@qmul.ac.uk

${ }^{b}$ Department of Chemical Engineering, Imperial College London, SW7 2AZ London, UK

${ }^{c}$ Tsinghua-Foxconn Nanoscience Research Center, Department of Physics, Tsinghua University, Beijing 100084, P. R. China

${ }^{d}$ Department of Chemical Engineering, University College London, Torrington Place, WC1E $7 J E$ London, UK

${ }^{e}$ State Key Laboratory of Photocatalysis on Energy and Environment College of Chemistry, Fuzhou University, Fuzhou 350116, P. R. China

${ }^{f_{S c h o o l}}$ of Materials Science and Engineering, Northwestern Polytechnical University, Xi'an 710072, P. R. China

$\dagger$ Electronic supplementary information (ESI) available. See DOI: 10.1039/ d0nr06139e
}

which is considered a promising renewable approach for solar energy conversion to clean and renewable chemical fuels that can help overcome the current energy crisis and environmental problems. $^{7,8}$ Since the pioneering work in the 1970s that demonstrated photocatalytic water splitting using titanium dioxide, ${ }^{9}$ multiple semiconductor materials have been developed as photoelectrodes for PEC water splitting, including $\mathrm{ZnO}, \mathrm{Fe}_{2} \mathrm{O}_{3}, \mathrm{WO}_{3}, \mathrm{BiVO}_{4}, \mathrm{SrTiO}_{3}, \mathrm{Ta}_{3} \mathrm{~N}$, and $\mathrm{C}_{3} \mathrm{~N}_{4} \cdot{ }^{10-15}$ However, the charge transfer and recombination at the interface of substrate/electrode and electrode/electrolyte significantly hinders their PEC water splitting efficiency. ${ }^{16}$ To address this, various strategies have been explored, such as doping, surface passivation, and substrate engineering. ${ }^{17-22}$

Carbon materials have shown to be able to address these interface-related issues by serving as conductive scaffolds, mediators, overlayers, and sacrificial underlayers, to accelerate electron transport and transfer and facilitate charge separation, hence improving their PEC performance. ${ }^{23-29}$ Hou et $a l{ }^{30}$ reported the use of $\mathrm{N}$-doped graphene as conductive nanoscaffolds for $\mathrm{g}-\mathrm{C}_{3} \mathrm{~N}_{4}$ photoanodes, which led to a remarkable photoelectrochemical performance for water oxidation, 
due to the multidimensional electron transport pathways, short charge transport time and distance, as well as effective separation and transport of photogenerated charge carriers. Particularly, carbon dots feature exceptional photoluminescence and photo-induced electron transfer properties, attracting increasing attention for their application in PEC. Carbon quantum dots applied on $\mathrm{ZnO}$ nanowire photoanodes was demonstrated to be able to enhance the visible-light absorption of $\mathrm{ZnO}$ nanowire photoanodes, thus improving incident photon-to-current efficiency by over 10 times. $^{31}$ Moradlou et $a .^{23}$ reported the use of carbon quantum dots as nano-scaffolds for $\alpha-\mathrm{Fe}_{2} \mathrm{O}_{3}$ growth, which led to a remarkable photoelectrochemical performance, due to the improvement in charge-transfer rate and suppression of electron-hole recombination derived from the increased hole-diffusion length in conducting nano-scaffold structure. Liang and coworkers $^{32}$ found that surface decoration of carbon quantum dots on $\mathrm{TiO}_{2}$ photoanodes could reduce the electron-hole recombination, attributed to the electron-trapping property of carbon quantum dots. Very recently, a N-doped carbon overlayer applied on $\alpha-\mathrm{Fe}_{2} \mathrm{O}_{3}$ photoanodes was shown to suppress interface charge recombination by passivating surface states, thus improving the photocurrent of hematite photoanodes. $^{25-27}$ Although carbon materials have played multiple roles in photoelectrodes, to the best of our knowledge, the use of carbon as underlayer in hematite photoanodes has not yet been reported.

Underlayers, introduced between substrate and semiconductor, can play a critical role in the PEC performance, by acting as a barrier for the suppression of electron back injection from fluorine-doped tin oxide (FTO) substrates to photoelectrodes, ${ }^{21,33}$ or as an electron transport layer for accelerating the electron transport from the semiconductor electrodes to the FTO substract, ${ }^{34}$ leading to higher charge separation yield. Additionally, underlayers have the potential to act as doping source for the semiconductor electrode material and improve the bulk conductivity. ${ }^{22,35}$ Underlayers can also help reduce the lattice mismatch between FTO and semiconductor films. ${ }^{36,37}$ Le Formal and co-workers ${ }^{36}$ demonstrated that $\mathrm{SiO}_{x}$ and $\mathrm{Nb}_{2} \mathrm{O}_{5}$ underlayers act as lattice strain buffers, improving crystallinity and uniformity of hematite films, and leading to less recombination at the interface.

Here we focus on the engineering of a carbon underlayer and exploration of its role in the structural and photoelectrochemical properties of hematite $\left(\alpha-\mathrm{Fe}_{2} \mathrm{O}_{3}\right)$ photoanodes. The carbon underlayer has been produced from carbon dots using biomass as raw material through a facile hydrothermal process. Carbon dots feature unique photoluminescence, electron trapping and electron transfer properties, ${ }^{38,39}$ are stable, low cost, nontoxic and easy to prepare. ${ }^{40}$ Hematite $\left(\alpha-\mathrm{Fe}_{2} \mathrm{O}_{3}\right)$ is considered one of the most exceptional photoanode candidates due to its nearly ideal bandgap, abundancy, nontoxicity, and chemical and photo stability. However, its intrinsic low conductivity, short hole diffusion length (2-4 nm), and poor carrier lifetime $\left(\sim 10^{-6} \mathrm{~s}\right)$, which lead to multiple charge recombination pathways, substantially limit the performance of hematite as photoanode. ${ }^{41,42}$ The as-synthesized carbon underlayer is expected to act as an electron transport layer and serve as dopant source for bulk $\mathrm{C}$ doping. The role of the carbon layer in the hematite photoanodes was carefully investigated by a combination of structural, compositional and electrochemical characterization techniques.

\section{Experimental}

\section{Materials}

Chitosan (medium molecular weight), ethylenediamine, iron (III) chloride hexahydrate $\left(\mathrm{FeCl}_{3} \cdot 6 \mathrm{H}_{2} \mathrm{O}, \geq 99.0 \%\right)$, sodium nitrate $\left(\mathrm{NaNO}_{3}, \geq 99.0 \%\right)$, and fluorine-tin-oxide $\left(\mathrm{FTO}, \mathrm{SnO}_{2} / \mathrm{F}\right.$, $\sim 13 \Omega \mathrm{sq}^{-1}$ ) glass substrates were purchased from SigmaAldrich. Hydrochloric acid ( $\mathrm{HCl}, 35 \mathrm{wt} \%)$ was provided by VWR Chemicals. Acetone and isopropanol were purchased from Fisher Chemicals. All reagents and solvents were used as received without further purification. All solutions were prepared with deionized water $\left(\sim 18.25 \mathrm{M} \Omega \mathrm{cm}, 25{ }^{\circ} \mathrm{C}\right)$ produced by a Milli-Q Element System (Millipore, Molsheim, France).

\section{Preparation of carbon dots-derived underlayer (UCDs)}

The carbon dots (CDs) were firstly synthesized by the hydrothermal carbonization of chitosan $(1.4 \mathrm{~g})$ and ethylenediamine $(580 \mu \mathrm{L})$ in $35 \mathrm{~mL}$ of water at $200{ }^{\circ} \mathrm{C}$ for 12 hours, followed by centrifugation at $20000 \mathrm{rpm}$ for $20 \mathrm{~min}$ and then filtration with standard syringe filters. FTO substrates were cut into $5 \mathrm{~cm} \times 1 \mathrm{~cm}$ pieces and ultrasonically cleaned by acetone, isopropanol, and water, sequentially. The clean FTO substrates with their conductive surface facing down were put into $50 \mathrm{~mL}$ autoclaves containing $20 \mathrm{~mL}$ of solutions with different volumes of CDs solution $(5 \mathrm{~mL}, 10 \mathrm{~mL}, 15 \mathrm{~mL}$, and $20 \mathrm{~mL}$, respectively), and then treated hydrothermally at $150{ }^{\circ} \mathrm{C}$ for 6 hours. The produced UCDs prepared with different volumes of CDs solution ( $5 \mathrm{~mL}, 10 \mathrm{~mL}, 15 \mathrm{~mL}, 20 \mathrm{~mL}$ ) are denoted as UCDs (5), UCDs (10), UCDs (15), UCDs (20), respectively, as summarized in Table 1.

\section{Fabrication of hematite photoanodes with UCDs (H/UCDs)}

H/UCDs photoanodes were fabricated by directly growing hematite $\left(\alpha-\mathrm{Fe}_{2} \mathrm{O}_{3}\right)$ nanorod arrays on the as-prepared UCDs. The obtained UCDs substrates were placed into a $50 \mathrm{~mL}$ of autoclave containing $0.1 \mathrm{M} \mathrm{FeCl}_{3}, 1 \mathrm{M} \mathrm{NaNO}_{3}$, and $60 \mu \mathrm{L}$ of $\mathrm{HCl}$, and thermally treated at $100{ }^{\circ} \mathrm{C}$ for 6 hours. A uniform yellow film of iron oxyhydroxide $(\mathrm{FeOOH})$ was obtained. The $\mathrm{FeOOH} / \mathrm{UCDs}$ were rinsed with distilled water, followed by

Table 1 Sample labelling

\begin{tabular}{lll}
\hline Synthesis conditions & $\begin{array}{l}\text { Carbon underlayers } \\
\text { (UCDs) }\end{array}$ & $\begin{array}{l}\text { Hematite/ } \\
\text { UCDs }\end{array}$ \\
\hline $5 \mathrm{~mL}$ CDs solution $+15 \mathrm{~mL}$ water & UCDs $(5)$ & H/UCDs(5) \\
$10 \mathrm{~mL}$ CDs solution $+15 \mathrm{~mL}$ water & UCDs $(10)$ & H/UCDs $(10)$ \\
$15 \mathrm{~mL}$ CDs solution $+15 \mathrm{~mL}$ water & UCDs $(15)$ & H/UCDs $(15)$ \\
$20 \mathrm{~mL}$ CDs solution $+15 \mathrm{~mL}$ water & UCDs $(20)$ & H/UCDs $(20)$
\end{tabular}


thermal annealing at $550{ }^{\circ} \mathrm{C}$ for two hours in air to produce hematite $(\mathrm{H}), \alpha-\mathrm{Fe}_{2} \mathrm{O}_{3}$. The same process was applied to all UCDs substrates, producing the photoanodes denoted as $\mathrm{H}$ / UCDs (5), H/UCDs (10), H/UCDs (15), and H/UCDs (20), respectively, as shown in Table 1 . A pristine hematite photoanode was also fabricated for comparison using the same procedure described above.

\section{Structural characterization}

Field emission scanning electron microscopy (FESEM, JEOL JSM-6301F) with an acceleration voltage of $5 \mathrm{kV}$ was used to study the morphology of the as-prepared UCDs and H/UCDs photoanodes. Atomic force microscopy (AFM, NanoWizard 4, JPK, Berlin, Germany) in tapping mode was used to image the as-prepared UCDs underlayer on FTO. Transmission electron microscopy (TEM, JEOL 2100 Plus) was employed to examine the CDs and photoanode samples at $200 \mathrm{kV}$ of electron beam energy. High-angle annular dark-field imaging- scanning transmission electron microscope (HAADF-STEM) images and electron energy loss spectroscopy (EELS) mapping was done with a probe aberration-corrected analytical electron microscope JEOL ARM200CF with operation voltage set at $200 \mathrm{kV}$ at the ePSIC facilities in Diamond Light Source. Copper grids with lacey carbon film were used as sample holder for the TEM and STEM-EELS measurements. The samples for TEM and STEM-EELS were prepared through a mechanical process reported elsewhere. ${ }^{16}$ Attenuated total reflectance Fourier Transform infrared spectroscopy (FTIR) spectra were recorded by using a Bruker Tensor 27 instrument in the range from $4000 \mathrm{~cm}^{-1}$ to $500 \mathrm{~cm}^{-1}$. UV-vis absorption spectra of all photoanodes were recorded with a UV-vis spectrophotometer (Lambda 950, PerkinElmer) equipped with an integrating sphere $(150 \mathrm{~mm}$ diameter sphere covered with Spectralon as the reflecting material, PerkinElmer) within a wavelength range of 350 to $800 \mathrm{~nm}$ and a step of $1 \mathrm{~nm}$. Photoluminescence spectra were investigated using a PerkinElmer LS55 spectrofluorimeter with Xenon pulsed flash lamp. X-ray diffraction (XRD) patterns were performed with a Panalytical Xpert Pro diffractometer with $\mathrm{Cu} \mathrm{K}_{\alpha}$ radiation (1.5418 ̊). X-ray photoelectron spectroscopy (XPS) analysis was carried out on a Thermo Scientific NEXSA XPS system with an $\mathrm{Al} \mathrm{K} \alpha \mathrm{X}$-ray source, and the data was analysed by Thermo Avantage software.

\section{Photoelectrochemical measurements}

Photocurrent measurements were carried out using a computer-controlled potentiostat (Gamry Instrument Interface 5000E) under both continuous and chopped illumination of $100 \mathrm{~mW}$ $\mathrm{cm}^{-2}$ with Xe light source (Newport $450 \mathrm{~W}$ ) calibrated using a Newport reference solar cell and meter (91550 V). Samples with a geometric area of $1 \mathrm{~cm}^{2}$ were illuminated from the back of the FTO substrate. Linear sweep voltammetry (LSV) measurements were conducted at a scan rate of $10 \mathrm{mV} \mathrm{s}^{-1}$ in the voltage range of $0.7 \mathrm{~V}-1.6 \mathrm{~V} v s$. RHE using a three-electrode system with a platinum foil as counter electrode and $\mathrm{Ag} / \mathrm{AgCl}$ (KCl sat.) as reference electrode. $1 \mathrm{M} \mathrm{NaOH}$ solution ( $\mathrm{pH}$ 13.6) was employed as electrolyte, previously purged with $\mathrm{N}_{2}(\mathrm{~g})$. Photoelectrochemical impedance spectroscopy (PEIS) measurements were conducted in a frequency range from 10 $\mathrm{kHz}$ to $0.1 \mathrm{~Hz}$, with an AC voltage amplitude of $10 \mathrm{mV}$ in the potential range from $0.8 \mathrm{~V}$ to $1.5 \mathrm{~V}$ vs. RHE with $0.05 \mathrm{~V}$ step size, using the same set up for the LSV tests. Intensity modulated photocurrent spectroscopy (IMPS) analysis was carried out using a potentiostat (IVIUM technology) under modulated illumination $\left(37.5 \mathrm{~mW} \quad \mathrm{~cm}^{2}\right)$ of $365 \mathrm{~nm}$ LED (ModuLightmodule, IVIUM technology) in a three-electrode configuration as that for LSV tests at varying potentials from $0.95 \mathrm{~V}$ to $1.35 \mathrm{~V} v s$. RHE with a step of $0.05 \mathrm{~V}$. A modulation of $10 \%$ in light intensity was applied, over a frequency range from $10 \mathrm{kHz}$ to $0.1 \mathrm{~Hz}$ at each potential step. PEIS and IMPS spectra were fitted using Zview software (Scribner).

\section{Results and discussion}

\section{Characterization of CDs and UCDs}

The carbon dots (CDs) synthesized to be used as underlayer (UCDs) exhibited a spherical shape and an average diameter of $4 \mathrm{~nm}$ (Fig. 1a). This correlates with the sizes usually obtained for CDs. ${ }^{43}$ In order to get a better understanding of their electronic structure and optical properties, UV-vis absorption spectroscopy and photoluminescence (PL) emission spectroscopy measurements were conducted (Fig. 1b). As expected, the CDs solution exhibits a strong, broad light absorption in the UV region at $\sim 280 \mathrm{~nm}$, with a tail extending into the visible range. The absorption at $\sim 280 \mathrm{~nm}$ is attributed to the $\pi \rightarrow \pi^{*}$ transition of $\mathrm{C}=\mathrm{C}$ bonds, while the visible light absorption is due to the $\mathrm{n} \rightarrow \pi^{*}$ transition of $\mathrm{C}=\mathrm{O}$ bonds. ${ }^{44}$ Their PL spectra show a strong emission at around $450 \mathrm{~nm}$ under $390 \mathrm{~nm}$ excitation wavelength, with a slight shift to larger wavelength
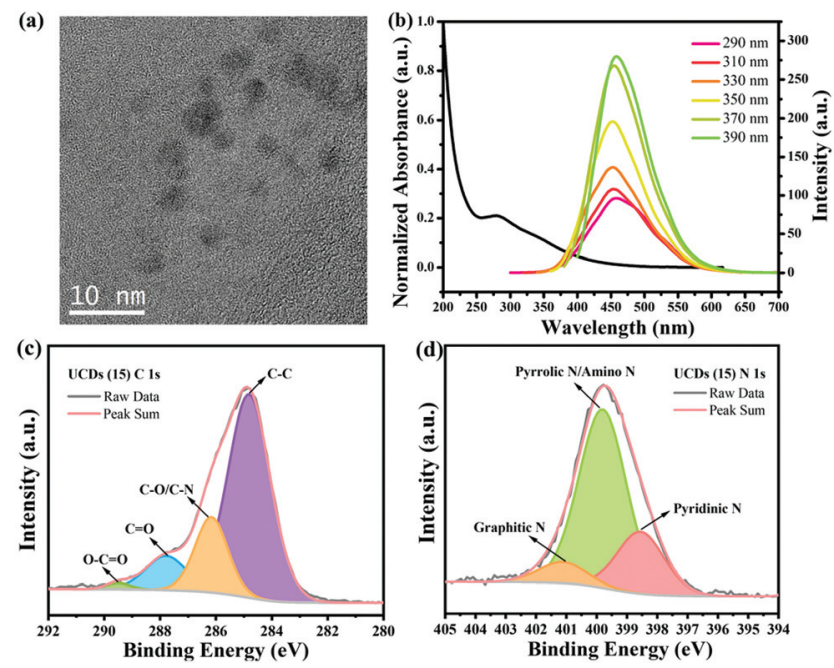

Fig. 1 (a) TEM image of the as-prepared CDs; (b) UV-vis spectrum and $\mathrm{PL}$ emission spectra at various excitation wavelengths for the as-prepared CDs aqueous solution; (c) C 1s and (d) N 1s core-level spectra of UCDs (15). 
values with increasing excitation wavelength. This also correlates with the behaviour typically is observed in CDs. ${ }^{43}$ FT-IR spectroscopy was performed to determine the functional groups present in the CDs and in the deposited UCDs (Fig. S1a $\dagger$ ). In the case of the CDs, stretching vibrations corresponding to $-\mathrm{O}-\mathrm{H} / \mathrm{N}-\mathrm{H}\left(\sim 3275 \mathrm{~cm}^{-1}\right), \mathrm{C}-\mathrm{H}\left(\sim 2925 \mathrm{~cm}^{-1}\right.$ and $\left.\sim 2855 \mathrm{~cm}^{-1}\right), \mathrm{C}=\mathrm{O}\left(\sim 1642 \mathrm{~cm}^{-1}\right)$, and $\mathrm{C}-\mathrm{O}\left(\sim 1003 \mathrm{~cm}^{-1}\right)$ bonds as well as $\mathrm{N}-\mathrm{H}$ bond bending vibrations $\left(\sim 1542 \mathrm{~cm}^{-1}\right)$, demonstrated the existence of oxygen-containing groups and amino groups at the CDs surface. ${ }^{45}$ In the case of the UCDs, the FTIR spectrum also features $\mathrm{C}-\mathrm{O}, \mathrm{C}-\mathrm{H}$ and $\mathrm{N}-\mathrm{H}$ functional groups, although the intensity of the bands is significantly lower, probably due to the small thickness of the UCDs layer deposited onto the FTO (Fig. S1a $\dagger$ ). The XPS survey scans of both CDs and UCDs (Fig. S1b†) also confirmed that the main components are $\mathrm{C}, \mathrm{N}$ and $\mathrm{O}$, consistent with the FT-IR data.

The contents of $\mathrm{C}, \mathrm{N}$ and $\mathrm{O}$ were determined to be $53.72 \%$, $15.39 \%$ and $30.89 \%$, respectively. The high-resolution XPS spectrum of the C 1s line (Fig. 1c) for the UCDs (15) sample was deconvoluted into five components: C-C $(284.8 \mathrm{eV}$, 68.49\%), C-O/C-N (286.2 eV, 19.18\%), $\mathrm{C}=\mathrm{O}(287.8 \mathrm{eV}$, $10.96 \%), \mathrm{O}-\mathrm{C}=\mathrm{O}(289.5 \mathrm{eV}, 1.37 \%)$, suggesting the existence of graphitic, aliphatic, nitrous, and oxygenated $\mathrm{C}^{45}$ In the case of the $\mathrm{N}$ 1s line (Fig. 1d), three types of $\mathrm{N}$ species were found, pyridinic $\mathrm{N}(\mathrm{C}-\mathrm{N}=\mathrm{C}, 398.6 \mathrm{eV}, 23.29 \%)$, pyrrolic N/amino $\mathrm{N}$ $(\mathrm{C}-\mathrm{N}-\mathrm{C} / \mathrm{N}-\mathrm{H}, 399.8 \mathrm{eV}, 68.49 \%)$, and graphitic $\mathrm{N}\left(\mathrm{N}-(\mathrm{C})_{3}, 401.1\right.$ $\mathrm{eV}, 8.22 \%),{ }^{46,47}$ existing both at the surface and inside the core structure. The presence of different types of $\mathrm{N}$ groups have shown to be beneficial for charge-transport properties. ${ }^{48}$

The deposition of the UCDs onto the FTO was also assessed by SEM and AFM (Fig. S2 $\dagger$ ), where a homogeneous film could be clearly seen covering the surface of the FTO substrate.

\section{Characterization of $\mathrm{H} / \mathrm{UCD}$ photoanodes}

Electron microscopy. The morphology of pristine hematite and H/UCDs photoanodes was investigated by SEM and TEM. As shown in Fig. 2a-e, hematite forms crystallites in the shape of nanorods, as previously reported. ${ }^{35}$ However, in the case of the H/UCDs photoelectrodes, the hematite nanorods exhibited
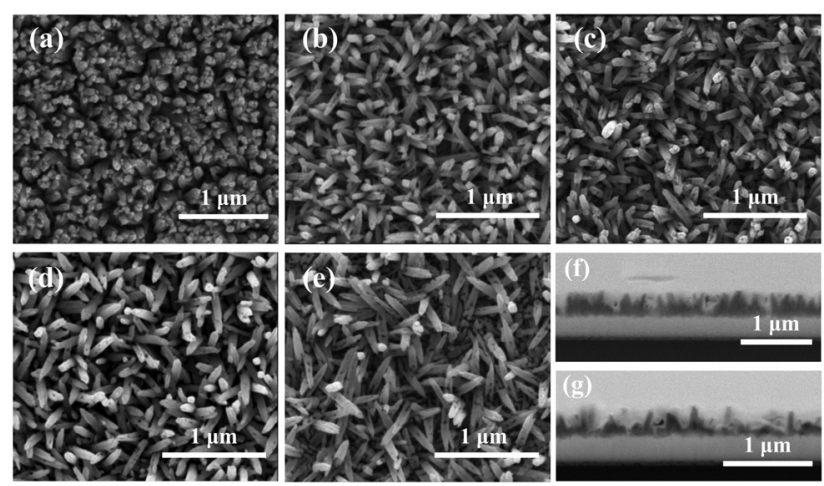

Fig. 2 SEM images of (a) pristine hematite, (b) H/UCDs (5), (c) H/UCDs (10), (d) H/UCDs (15), (e) H/UCDs (20); cross-section SEM images of the (f) pristine hematite and (g) H/UCDs (10) photoanodes. a somewhat random alignment, attributed to an increase in the surface roughness of the substrate after the deposition of the UCDs layer, accompanied by a shortening in the size of the nanorods (Fig. $2 \mathrm{f}-\mathrm{g}$ and $\mathrm{S} 3 \dagger$ ). This becomes especially noticeable for the sample with the highest concentration of CDs, H/ UCDs (20). This observation is consistent with previously reported hematite photoanodes using underlayers. ${ }^{16}$

The chemical composition and electronic structure of pristine hematite and H/UCDs (15) photoanodes were studied by HAADF-STEM with EELS mapping (Fig. 3). As expected, the individual $\mathrm{C}, \mathrm{O}, \mathrm{Fe}$, and combined $\mathrm{Fe}+\mathrm{O}+\mathrm{C}$ RGB EELS mapping images revealed that pristine hematite and the $\mathrm{H}$ / UCDs (15) are mostly composed of Fe and O. Although $\mathrm{C}$ is observed for both samples, the weak and randomly distributed C signal detected in the pristine hematite is attributed to environmental adventitious carbon as a consequence of being exposed to air (Fig. 3a). In contrast, the strong and more uniform distribution of carbon along the edges of the H/UCDs (15) nanorods (Fig. 3b) suggests that in this case the C comes most likely from the UCDs layer. We believe this $\mathrm{C}$ may have been introduced in the hematite via decomposition and volatilization of the carbon underlayer during the transformation of $\mathrm{FeOOH}$ into $\alpha-\mathrm{Fe}_{2} \mathrm{O}_{3}$ (hematite). The derived $\mathrm{C}$ overlayer on hematite is expected to contribute to suppressing surface charge recombination by passivating surface states, thus boosting the photocurrent of hematite photoanodes. ${ }^{25-27}$

Fig. $3 \mathrm{c}$ and $\mathrm{d}$, and e present the corresponding EELS spectra of the $\mathrm{C} \mathrm{K}$ edge, $\mathrm{O} \mathrm{K}$ edge, and $\mathrm{Fe}_{2,3}$ edge for pristine hematite and H/UCDs (15). The corresponding zero-loss peaks of EELS spectrum were provided in Fig. S4. $\dagger$ In the case of the $\mathrm{H} / \mathrm{UCDs}$ (15), the $\mathrm{C}$ signal displays a first peak at $285 \mathrm{eV}$, induced by $\pi^{*}$ molecular orbital transitions due to the presence of $\mathrm{sp}^{2}$ bonding, and a second peak at $290 \mathrm{eV}$ induced by transitions to $\sigma^{*}$ orbitals. In the case of pristine hematite, four peaks (a-d) were identified (Fig. 3d) for the O-K edge signal. Peak a is related to the hybridization of the $\mathrm{O} 1 \mathrm{~s}$ to $2 \mathrm{p}$ with the Fe 3d orbital, while peak $b$ is associated with the hybridization between the $\mathrm{O} 2 \mathrm{p}$ orbital and the Fe $4 \mathrm{~s}$ and $4 \mathrm{p}$ orbitals. Peaks $c$ and $d$ are caused by the scattering of the third and the first oxygen coordination shells. ${ }^{49-51}$ There are no significant differences between the $\mathrm{O}-\mathrm{K}$ edge spectrum of pristine hematite and H/UCDs (15), indicating similar oxygen coordination and electronic environment for both samples. The $\mathrm{L}$ edge spectrum of metal cations can be used to probe their ionization state through the analysis of the relative position and intensity of individual $\mathrm{L}_{3}$ and $\mathrm{L}_{2}$ edges. ${ }^{49,51,52}$ The Fe $\mathrm{L}_{3}$ peak of the $\mathrm{H}$ / UCDs (15) exhibited a chemical shift of $0.9 \mathrm{eV}$ toward lower energy compared to that of the pristine hematite (Fig. 3e). The separation between $\mathrm{L}_{3}$ and $\mathrm{L}_{2}$ peaks increases from $12.9 \mathrm{eV}$ for the pristine hematite to $13.4 \mathrm{eV}$ for H/UCD (15). In addition, the intensity ratio of $\mathrm{L}_{3} / \mathrm{L}_{2}$ is $c a .5 .2$ for the H/UCDs (15), whereas the value for pristine hematite is $c a$. 5.5. These results suggest a change in the ionization state of the $\mathrm{Fe}$ in the $\mathrm{H}$ / UCDs (15), which has previously been attributed to either partial change in the oxidation state from $\mathrm{Fe}^{3+}$ to $\mathrm{Fe}^{2+}$ due to the $\mathrm{Fe}-\mathrm{C}$ coordination or the formation of oxygen 

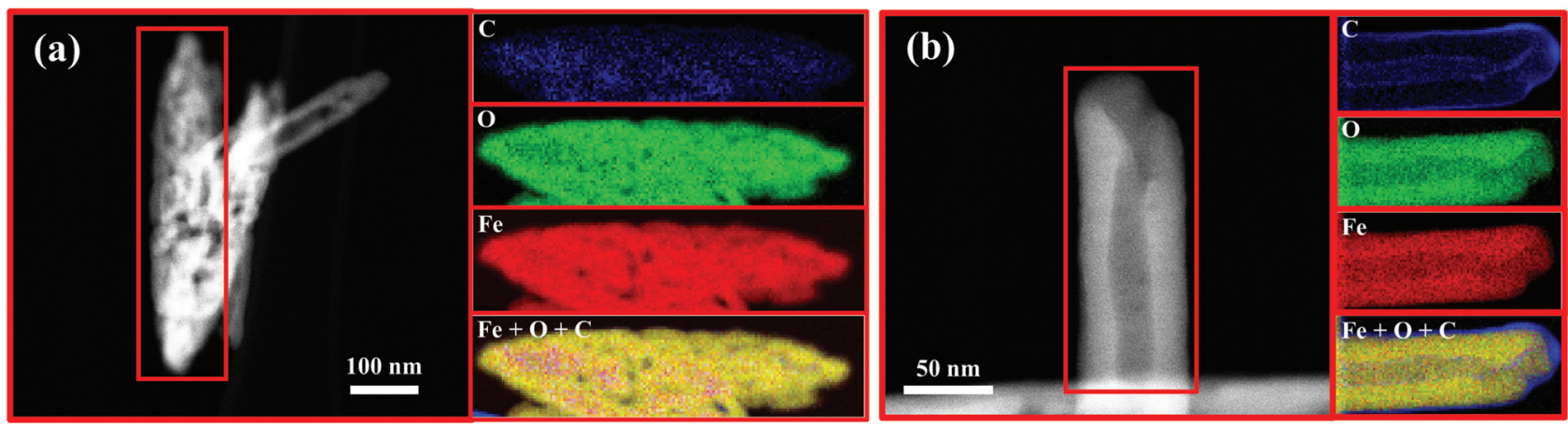

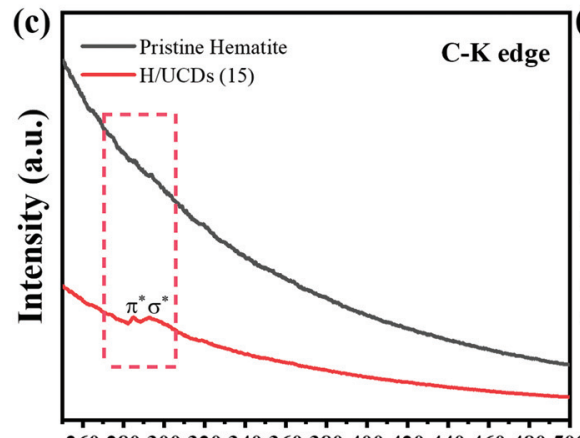

260280300320340360380400420440460480500 Energy Loss (eV)
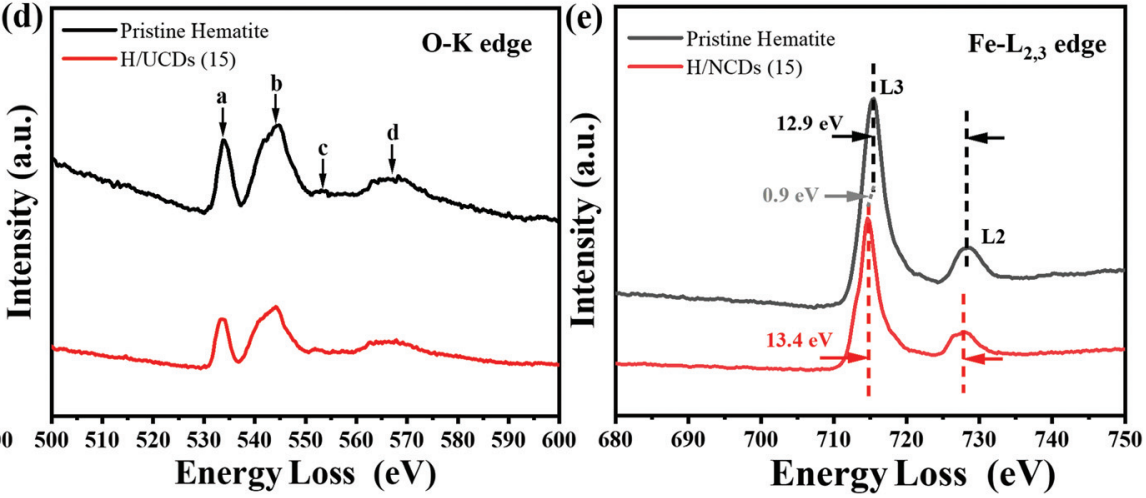

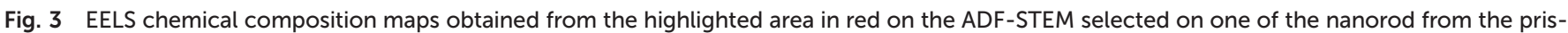

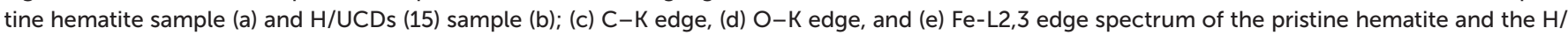
UCDs (15).

vacancies. ${ }^{49,51,52}$ Due to the polaron hopping mechanism in hematite, the existence of $\mathrm{Fe}^{2+}$ could significantly increase the conductivity of the material. ${ }^{53,54}$

XRD, XPS and UV-visible absorption spectroscopy. Pristine hematite and H/UCDs samples displayed similar XRD patterns, with reflections corresponding to hematite, with a structure consisting of a dense arrangement of $\mathrm{Fe}^{3+}$ ions in octahedral coordination with oxygen $\mathrm{O}^{2-}$ ions in hexagonal closest-packing $\left(\alpha-\mathrm{Fe}_{2} \mathrm{O}_{3}\right.$ : JCPDS \#33-0664), and tin oxide from FTO substrates ( $\mathrm{SnO}_{2}$ : JCPDS \#46-1088) (Fig. S5a $\dagger$ ). Preferred orientation along the [110] axis was observed, in good agreement with the nanorod morphology seen in TEM and SEM.

The XPS survey spectra (Fig. S4b $\dagger$ ) for pristine hematite and $\mathrm{H} / \mathrm{UCD}$ samples indicate that all of the prepared photoanode surfaces consist of $\mathrm{Fe}, \mathrm{O}$ and $\mathrm{C}$. The presence of $\mathrm{C}$ in the XPS spectrum of the pristine hematite is attributed to adventitious carbon contamination. For the Fe 2p XPS line (Fig. 4a), two dominant peaks corresponding to $\mathrm{Fe} 2 \mathrm{p}_{1 / 2}(\sim 724.3 \mathrm{eV})$ and $\mathrm{Fe}$ $2 \mathrm{p}_{3 / 2}(\sim 711.1 \mathrm{eV})$ are observed, accompanied by their shakeup satellite peaks centered at $718.7 \mathrm{eV}$ and $732.9 \mathrm{eV}\left(\mathrm{Fe} \mathrm{2} \mathrm{p}_{1 / 2}\right.$, sat and $\mathrm{Fe} 2 \mathrm{p}_{3 / 2}$, sat), confirming the presence of $\mathrm{Fe}^{3+}$ as $\mathrm{Fe}_{2} \mathrm{O}_{3}$.

55 Importantly, a careful inspection of $\mathrm{Fe} 2 \mathrm{p}$ spectrum reveals that a red shift $(0.5 \mathrm{eV})$ of the $\mathrm{Fe} 2 \mathrm{p}_{1 / 2}$ satellite towards lower energy (Fig. 4a) for the H/UCDs samples relative to that of the pristine hematite, suggesting the existence of $\mathrm{Fe}^{2+}$ or $\mathrm{Fe}-$ $\mathrm{C}$ bonds, which is consistent with the EELS data. ${ }^{56-58}$ The $\mathrm{O} 1 \mathrm{~s}$ spectrum (Fig. 4b) exhibited a main line at $530.0 \mathrm{eV}$ with a
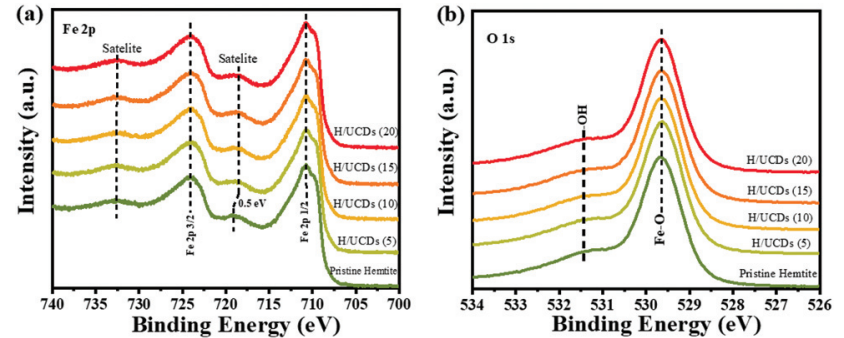

Fig. 4 (a) High resolution XPS of Fe $2 p$ line, (b) High resolution XPS of $\mathrm{O}$ 1s line for pristine hematite and H/UCDs photoanodes.

shoulder peak at $531.5 \mathrm{eV}$ for all samples, corresponding to the coordination of $\mathrm{Fe}-\mathrm{O}$ bond and surface hydroxyl species, respectively. ${ }^{55,59}$

Pristine hematite and H/UCDs show similar optical absorbance edge, from $\sim 600$ to $\sim 400 \mathrm{~nm}$, indicating the introduction of UCDs does not have a significant effect on the UV-vis absorption range of hematite photoanodes (Fig. S6a †). However, the absorbance intensity for the H/UCDs samples was lower than that of the pristine hematite, this being particularly notable for the H/UCDs (20) sample, which can be explained by the fact that the shortened and obliquely oriented $\mathrm{H} / \mathrm{UCD}$ nanorods produce a reduction in the light absorption relative to the thicker and vertically oriented pristine hematite sample, as already predicted from the SEM images of the $\mathrm{H}$ / UCDs samples. ${ }^{60}$ The Tauc plots for an indirect and a direct 
band gap are shown in Fig. S6b and S6c, $\uparrow$ respectively, showed a band gap of around $1.73 \mathrm{eV}$ for the indirect transition and around $1.80 \mathrm{eV}$ for the direct transition with no significant changes among the different samples.

\section{Photoelectrochemical (PEC) performance and mechanism studies}

Photocurrent densities. A low photocurrent response was observed for the pristine hematite (Fig. 5a). This was anticipated as our pristine hematite was synthesised at $550{ }^{\circ} \mathrm{C}$, and temperatures above $800{ }^{\circ} \mathrm{C}$ are needed to achieve reasonable photocurrent values, usually attributed to the doping of hematite with Sn from the FTO. ${ }^{54,61-64}$ We made the decision to prepare our hematite below the decomposition temperature of the FTO to avoid any additional factors that could mask the effect of the UCDs. All the hematite photoanodes modified with UCDs (H/UCDs) showed increased photocurrent densities relative to pristine hematite. The best performing photoanode was H/UCDs (15). In addition, the photoelectrodes exhibited a prompt and reproducible photocurrent response (Fig. 5b), corresponding to the on-off illumination cycles with the same photocurrent densities as those under continuous irradiation, further evidencing the enhancement in PEC performance by the introduction of UCDs. In order to elucidate why H/UCDs (15) exhibited the best performance, Mott-Schottky, PEIS and IMPS were conducted.

Mott-Schottky measurements. In order to understand the enhancement of the photocurrent response for the H/UCDs photoanodes, Mott-Schottky measurements were conducted (Fig. S7†). The flat band $\left(E_{\mathrm{fb}}\right)$ and carrier density $\left(N_{\mathrm{d}}\right)$ determined from the intercept and slope by extrapolation of the linear variation part of $1 / C^{2}$ against potential $E$ (Fig. $5 \mathrm{c}$ ) according to the eqn (S1) and (S2) (ESI $\dagger$ ), are given in Table S1. $\dagger$ The results showed an increased $N_{\mathrm{d}}$ for the H/UCDs samples. The
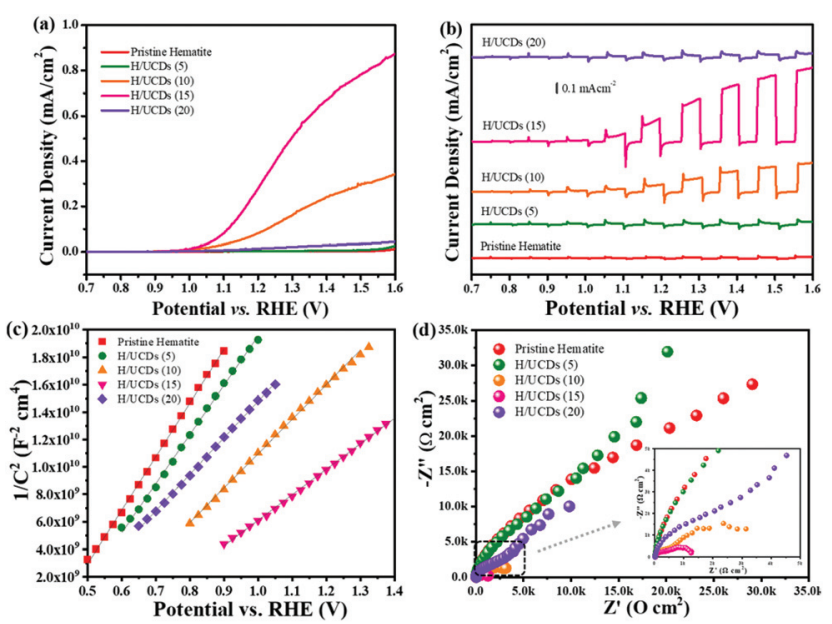

Fig. 5 LSV curves under (a) light and (b) chopped conditions for pristine hematite and H/UCDs photoanodes. (c) Linear fitting of Mott-Schottky plots for hematite and H/UCDs samples. (d) Nyquist plots of hematite and H/UCDs samples at $1.25 \mathrm{v}$ vs. RHE and inset shows the enlarged view of circled area. maximum $N_{\mathrm{d}}\left(2.30 \times 10^{18}\right)$ achieved by H/UCDs $(15)$ is about two times more than that of the pristine hematite $\left(1.09 \times 10^{18}\right)$, which we attribute to the partial change in the oxidation state of $\mathrm{Fe}$ from $\mathrm{Fe}^{3+}$ to $\mathrm{Fe}^{2+}$, due to $\mathrm{Fe}-\mathrm{C}$ coordination or the formation of vacancies, as revealed by EELS and XPS. ${ }^{63}$ In addition, the existence of UCDs as underlayer is expected to contribute to the back electrons collection, which yields a boost in the bulk charge separation, thus leading to the increased $N_{\mathrm{d}}$ for H/UCDs samples.

The $E_{\mathrm{fb}}$ value for the H/UCDs samples shifts to higher potentials, i.e. from $0.43 \mathrm{~V} v$ s. RHE for pristine hematite to $0.67 \mathrm{~V} v s$. RHE for H/UCDs (15). This anodic shift of $E_{\mathrm{fb}}$ with an increased $N_{\mathrm{d}}$ is unexpected, as an increase in $N_{\mathrm{d}}$ usually yields a negative shift of $E_{\mathrm{fb}}$ by raising the Fermi level $\left(E_{\mathrm{f}}\right)$ closer to the conduction band $\left(E_{\mathrm{CB}}\right)$, thus promoting band bending to accelerate the charge separation. This phenomenon has been previously reported for other doping systems with enhanced photocurrents. However, it has not been satisfactorily explained yet. ${ }^{65-67}$ The Mott-Schottky measurements at different frequencies ( $3 \mathrm{kHz}$ and $5 \mathrm{kHz}$ ), further confirmed these observations (Fig. S8 and Table $\mathrm{S} 2 \dagger$ ) and attributed the enhanced PEC performance for the H/UCDs photoanodes to the increased $N_{\mathrm{d}}$.

PEIS study. PEIS measurements under illumination were performed to study the mechanism by which the hematite photocurrent is enhanced by the presence of the carbon underlayer. The obtained Nyquist plots at $1.25 \mathrm{~V} v s$. RHE are shown in Fig. 5d. The semicircle in high-frequency range is associated with surface charge recombination by trapping states, while the impedance feature in the low-frequency range can be related to the interfacial charge transfer. ${ }^{68}$ The H/UCDs samples (except for the H/UCDs (5)) show a remarkably reduced radius for both impedance featured semicircles relative to that of the pristine hematite, with the smallest radius achieved by the H/UCDs (15). A series of PEIS measurements in the potential range $0.80-1.5 \mathrm{~V} v s$. RHE were conducted. The results were fitted based on a two-RC-unit equivalent circuit proposed by Klahr et al., where the surface state is assumed to serves as the hole-trapping center for charge separation and transfer, which is often employed for hematite photoanodes. ${ }^{69}$ In this equivalent circuit (inset Fig. S8a $\dagger$ ), the series resistance $\left(R_{\mathrm{S}}\right)$ is associated with the FTO substrate and the electrolyte, the trapping resistance $\left(R_{\text {trap }}\right)$ is caused by the surface states where the recombination of electron-hole pairs happens, the charge-transfer resistance $\left(R_{\mathrm{ct}}\right)$ is the resistance at the semiconductor-liquid interface when charge transfer happens. The two capacitors are bulk capacitor $\left(C_{\text {bulk }}\right)$ and surface states capacitor $\left(C_{\mathrm{ss}}\right)$, respectively. The Nyquist plot for the photoanode H/UCDs (15) can be fitted using this equivalent circuit without the need of constant phase elements (Fig. S9a $\dagger$ ). The fitting results were plotted as function of potentials in Fig. S9b-e, $\uparrow$ and the values at $1.25 \mathrm{~V} v s$. RHE were collected in Table S1. $\dagger$ All the H/UCDs photoanodes (except H/UCDs (5)) exhibited lower values of $R_{\text {trap }}$ for the H/UCDs electrodes $(0.8 \mathrm{~V}$ to $1.5 \mathrm{~V} v s$. RHE) compared to pristine hematite, suggesting a reduced charge recombination. The H/UCDs (10) and H/UCDs 
(15) photoelectrodes exhibit gradual decrease in $R_{\text {trap }}$ with increasing voltage below $1.25 \mathrm{~V}$ vs. RHE followed by a sustained growth, evidencing that the charge transfer increases at higher potentials, consistent with the increment of the associated photocurrent response. In contrast, the rest of the samples show a constant increase in $R_{\text {trap }}$ over the measured potential range, which has also been observed in previous studies, although still not fully understood. ${ }^{69,70}$ A similar situation can be observed for the $R_{\mathrm{ct}}$ values (Fig. S9c $\dagger$ ). Starting from 1.05 V vs. RHE, the $R_{\mathrm{ct}}$ of H/UCDs (10) and H/UCDs (15) decrease with increasing voltage, and after reaching a minimum, $R_{\mathrm{ct}}$ starts to increase. The $R_{\mathrm{ct}}$ of the pristine hematite, H/UCDs (5), and H/UCDs (20) show opposite trend. The lowest $R_{\mathrm{ct}}$ of H/UCDs (15) at $1.25 \mathrm{~V} v s$. RHE is around two orders of magnitude lower than that of the pristine hematite, indicating a promoted charge transfer from surface states to electrolyte. The higher $C_{\text {bulk }}$ values (Fig. S9d $\dagger$ ) found for $\mathrm{H} /$ UCDs samples except for the case of H/UCDs (5) indicates an increased carrier concentration, in good agreement with the Mott-Schottky results. From Fig. S9e, $\dagger$ a higher $C_{\mathrm{ss}}$ is observed for $\mathrm{H} / \mathrm{UCD}$ samples including the H/UCDs (5) relative to that of the pristine hematite. The surface states of metal oxides have been widely considered as electron-hole recombination centres. However, recent studies showed that they could also serve as hole collectors and facilitate charge accumulation and transfer. ${ }^{71-74}$ Therefore, the higher $C_{\mathrm{ss}}$ values for the H/UCDs photoelectrodes indicate more holes were trapped to participate in the water oxidation process. From the PEIS study, it can be concluded that the superior PEC performance for $\mathrm{H} /$ UCDs samples is the result of the reduced $R_{\text {trap }}$ and $R_{\text {ct }}$ along with the increased $C_{\mathrm{ss}}$ all of which facilitate the charge separation and transfer.

IMPS investigation. IMPS is a useful tool for an in-depth exploration of the surface kinetics of charge transfer and recombination. ${ }^{65,75-77}$ The theory for the IMPS has been thoroughly discussed and demonstrated to be suitable for hematite photoanodes. ${ }^{68,75}$ Fig. 6a compares the IMPS spectra at $1.25 \mathrm{~V} v s$. RHE for all samples, and the full IMPS dataset for hematite and H/UCDs (15) from 0.95 to $1.35 \mathrm{~V} v s$. RHE are displayed in Fig. $6 \mathrm{~b}$ and c. The IMPS spectrum consisted of two semicircles in the complex plane located in the first and the fourth quadrants corresponding to the low and high frequency region, respectively. The high-frequency arc in the fourth quadrant reflects the attenuation of the PEC system caused by the series resistance and capacitances, the frequency at the minimum of which can be related to the diffusion lifetime of photogenerated electrons from the electrode to the back contact, ${ }^{76,78}$ whereas the low-frequency arc, also called the recombination semicircle, is associated with the charge transfer and recombination process at the electrode-liquid interface. For the pristine hematite, the two semicircles appear to form a closed circle, which suggests a poor charge transfer and notable recombination process at the pristine hematite surface, as expected. In comparison, the H/UCDs (15) showed distinctly separated semicircles, indicating a better charge separation and transfer efficiency. Furthermore, H/UCDs (15) recombination semicircles exhibited a significant contraction as the potential is increased, implying an improved charge separation and transfer efficiency with the increase of potentials, while the recombination semicircles of the rest samples show slight changes with the potentials.

The rate constants, $k_{\mathrm{ct}}$ (hole transfer rate constant) and $k_{\mathrm{rec}}$ (surface charge recombination rate constant), the overall charge transfer efficiency (CTE), as well as the diffusion lifetime of the photogenerated electrons carriers $\left(\tau_{\mathrm{d}}\right)$ at various potential determined from the IMPS characteristic constants (the frequencies at the minimum and maximum of the semicircles, the low- and high-frequency intercepts) are shown in Fig. 7. Detailed calculation steps can be found elsewhere. ${ }^{68,75}$ The $k_{\mathrm{ct}}$ values for all samples (Fig. 7a) increase with increasing potentials. The H/UCDs (10) and H/UCDs (15) exhibit comparable $k_{\text {ct }}$ values, which are much higher than the rest of the samples. The $k_{\mathrm{ct}}$ value of H/UCDs (15) at $1.25 \mathrm{~V} v s$. RHE. is almost four times than that of the pristine hematite, indicating an improved hole transfer at its electrode/electrolyte interface. The $k_{\text {rec }}$ values for H/UCDs (10) and H/UCDs (15) are found decrease with the increase of the potential, whereas the $k_{\text {rec }}$ values for H/UCDs (5), H/UCDs (20) and hematite remain constant with similar values as a result of Fermi level pinning effects (Fig. 7b). The H/UCDs (10) and H/UCDs (15) samples show a reduced $k_{\text {rec }}$ compared to that of pristine hematite at potentials above $1.15 \mathrm{~V}$ and $1.05 \mathrm{~V}$ vs. RHE, respectively. The
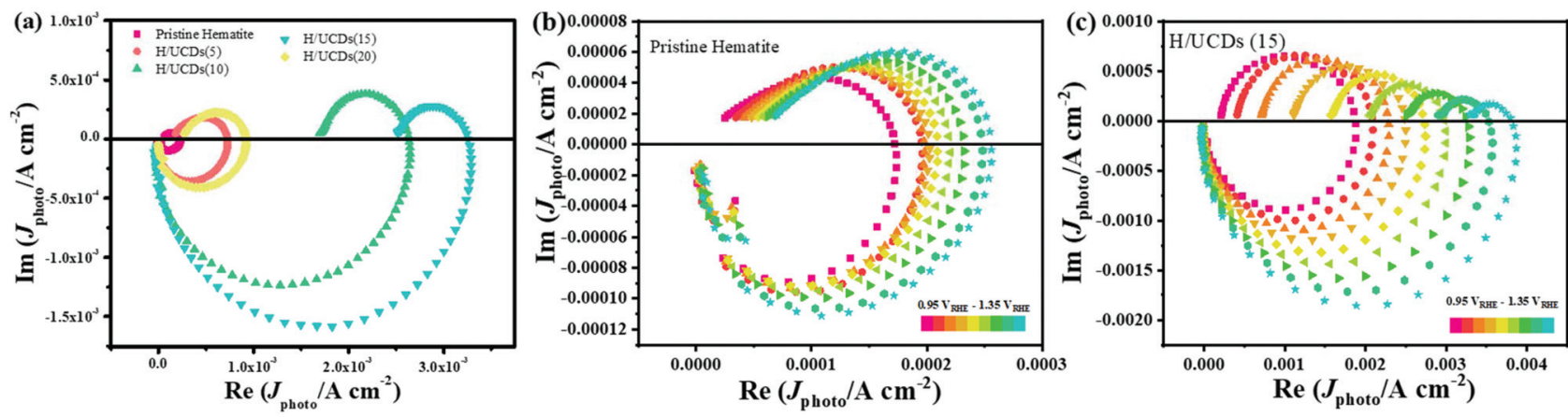

Fig. 6 IMPS plots for (a) pristine hematite and H/UCDs photoelectrodes; (b) pristine hematite; (c) H/UCDs (15). 

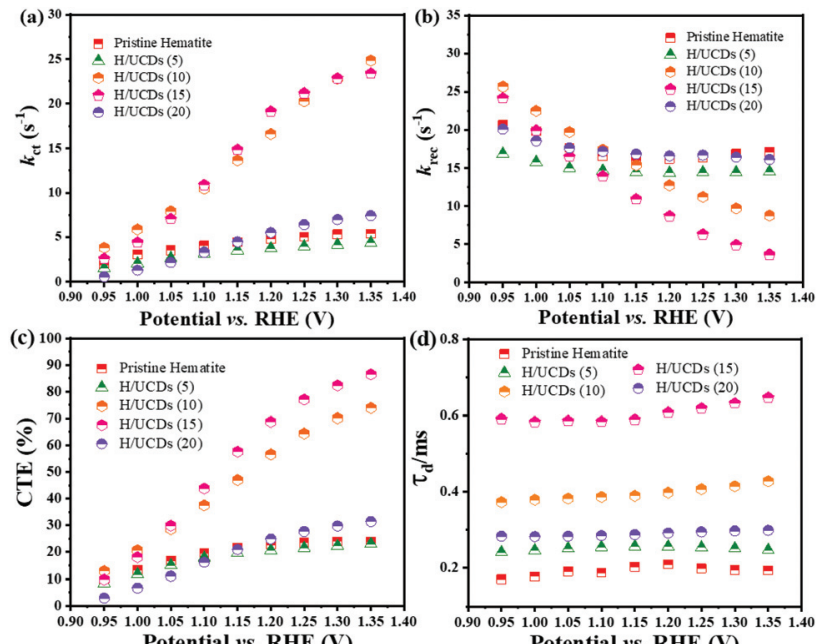

Potential vs. RHE (V)

Potential vs. RHE (V)
Fig. 7 (a) $k_{\mathrm{tr}}$, (b) $k_{\mathrm{rec}}$ (c) charge transfer efficiency (CTE), and (d) diffusion lifetime of the photogenerated charge carriers calculated from IMPS plots.

reduced $k_{\text {rec }}$ for H/UCDs (10) and H/UCDs (15) indicate a lower surface recombination at the surface state. Note that the $\mathrm{H} /$ UCDs (15) has lower $k_{\text {rec }}$ but similar $k_{\text {ct }}$ compared to H/UCDs (10). This suggests that the further improved photocurrent of $\mathrm{H} / \mathrm{UCDs}$ (15) relative to that of H/UCDs (10) is primarily attributed to the decrease in charge recombination. The H/UCDs (20) shows a similar $k_{\text {rec }}$ as that of the pristine hematite, whereas the H/UCDs (5) exhibits a lower $k_{\text {rec }}$. This phenomenon suggests that is the $k_{\mathrm{ct}}$, rather than $k_{\text {rec }}$, one of the main factors contributing to the increase in photocurrent for the $\mathrm{H}$ / UCDs (20). The CTE presented in Fig. 7c further demonstrates the improved charge transfer process for H/UCDs (10) and H/ UCDs (15) as a result of their higher $k_{\text {ct }}$ and lower $k_{\text {rec }}$. All the $\mathrm{H} / \mathrm{UCDs}$ photoelectrodes show a distinct enhancement in $\tau_{\mathrm{d}}$ as compared to the pristine hematite, i.e. the $\tau_{\mathrm{d}}$ of H/UCDs (15) (Fig. 7d). A longer $\tau_{\mathrm{d}}$ means an improved back electron collection and a less recombination during the transfer of electrons to the substrate at the substrate-electrode interface, which allows more holes to be transferred to the electrode surface for water oxidation, hence contributing to the photocurrent enhancement. $^{76}$ Thus, the IMPS results further reveal that the improved photocurrent for H/UCDs samples is due to the enhanced $k_{\mathrm{ct}}$, reduced $k_{\mathrm{rec}}$, as well as extended $\tau_{\mathrm{d}}$.

\section{Conclusions}

Carbon underlayers produced from biomass - derived carbon dots through a facile hydrothermal process were introduced in hematite photoanodes, in order to enhance the photoresponse of hematite for application in solar-driven water splitting. The formation of this carbon underlayer in combination with a hematite thin film photoelectrode resulted in a remarkable increase in photocurrent density $\left(0.35 \mathrm{~mA} \mathrm{~cm}^{-2}\right.$ at $1.23 \mathrm{~V} v s$. RHE) compared to pristine hematite. The presence of the carbon underlayer contributes to the back electron collection and transfer, as all the H/UCDs photoelectrodes exhibited longer $\tau_{\mathrm{d}}$ than pristine hematite. Among all the H/UCDs, H/ UCDs (15) exhibited the best performance. The promoted back electron collection led to an increase in the carrier concentration in bulk and hole accumulation in surface states for water oxidation. This could be confirmed by the higher $C_{\text {bulk }}$ and $C_{\mathrm{ss}}$ values of $\mathrm{H} / \mathrm{UCDs}$ photoelectrodes relative to the pristine hematite.

The presence of UCDs also led to the formation of a carbon overlayer via partial decomposition and volatilization of the UCDs during the transformation of $\mathrm{FeOOH}$ into hematite, as evidenced by the EELS mapping. The carbon overlayer has been demonstrated to be able to help passivate surface state thus promoting the hole transfer and suppressing charge recombination, which correlates well with the remarkable $k_{\mathrm{ct}}$ and $k_{\text {rec }}$ for H/UCDs (10) and H/UCDs (15). Finally, the presence of UCDs and its partial decomposition and volatilization induce the incorporation of $\mathrm{C}$ in hematite, resulting in partial change in the oxidation state from $\mathrm{Fe}^{3+}$ to $\mathrm{Fe}^{2+}$ by $\mathrm{Fe}-\mathrm{C}$ coordination or oxygen vacancy, as indicated by the EELS and XPS analysis. The oxidation state change can improve the bulk conductivity through the polaron hopping mechanism, in good agreement with the improved $N_{\mathrm{d}}$ indicated by Mott-Schottky measurement. This work has shown for the first time a remarkable enhancement of the hematite photocurrent density through the application of a carbon underlayer between FTO and hematite. The triple role of the carbon underlayer that (1) improved the FTO/hematite interfacial properties, (2) provided a carbon source for the formation of an overlayer, and (3) promoted the reduction of some $\mathrm{Fe}^{3+}$ to $\mathrm{Fe}^{2+}$, led to a significant enhancement of bulk and interfacial charge transfer dynamics in hematite, and hence a remarkable improvement in the photoactivity.

\section{Conflicts of interest}

There are no conflicts to declare.

\section{Acknowledgements}

ABJ would like to thank EPSRC (EP/P031323/1) for the financial support. QG and HL acknowledge the CSC scholarship. YF and XW thank the Royal Society for the Advanced Newton Fellowship (NAF $\backslash$ R1 $\backslash 180198), \quad$ National Natural Science Foundation of China (21703040 and 21861130353) for the financial support. QR acknowledges the UCL Dean's prize and China CSC scholarship. QR and JT acknowledge the financial supports from UK EPSRC (EP/N009533/1) and the Leverhulme Trust (RPG-2017-122). We thank Dr Russell Bayley and Dr Vicente Araullo-Peters for the help of material characterization and the Diamond Light Source for access and support in use of the electron Physical Science Imaging Centre (Instrument E01 proposal MG22447). 


\section{References}

1 Q. Ma, Y. Yu, M. Sindoro, A. G. Fane, R. Wang and H. Zhang, Adv. Mater., 2017, 29, 1605361.

2 J. Wang, P. Nie, B. Ding, S. Dong, X. Hao, H. Dou and X. Zhang, J. Mater. Chem. A, 2017, 5, 2411-2428.

3 J. Briscoe, A. Marinovic, M. Sevilla, S. Dunn and M. Titirici, Angew. Chem., Int. Ed., 2015, 54, 4463-4468.

4 R. Sekiya, Y. Uemura, H. Murakami and T. Haino, Angew. Chem., Int. Ed., 2014, 53, 5619-5623.

5 Y. Si, X. Wang, C. Yan, L. Yang, J. Yu and B. Ding, Adv. Mater., 2016, 28, 9512-9518.

6 L. Wang, Q. Zhang, S. Chen, F. Xu, S. Chen, J. Jia, H. Tan, H. Hou and Y. Song, Anal. Chem., 2014, 86, 1414-1421.

7 Y. Tachibana, L. Vayssieres and J. R. Durrant, Nat. Photonics, 2012, 6, 511.

8 M. Grätzel, Nature, 2001, 414, 338.

9 A. Fujishima and K. Honda, Nature, 1972, 238, 37.

10 X. Yang, A. Wolcott, G. Wang, A. Sobo, R. C. Fitzmorris, F. Qian, J. Z. Zhang and Y. Li, Nano Lett., 2009, 9, 2331-2336.

11 A. Kay, I. Cesar and M. Grätzel, J. Am. Chem. Soc., 2006, 128, 15714-15721.

12 W. Li, P. Da, Y. Zhang, Y. Wang, X. Lin, X. Gong and G. Zheng, ACS Nano, 2014, 8, 11770-11777.

13 T. W. Kim and K.-S. Choi, Science, 2014, 343, 990-994.

14 Y. Li, T. Takata, D. Cha, K. Takanabe, T. Minegishi, J. Kubota and K. Domen, Adv. Mater., 2013, 25, 125-131.

15 J. Bian, Q. Li, C. Huang, J. Li, Y. Guo, M. Zaw and R.-Q. Zhang, Nano Energy, 2015, 15, 353-361.

16 P. Tang, H. Xie, C. Ros, L. Han, M. Biset-Peiro, Y. He, W. Kramer, A. P. Rodriguez, E. Saucedo, J. R. GalanMascaros, T. Andreu, J. R. Morante and J. Arbiol, Energy Environ. Sci., 2017, 10, 2124-2136.

17 W. J. Jo, J. W. Jang, K. j. Kong, H. J. Kang, J. Y. Kim, H. Jun, K. Parmar and J. S. Lee, Angew. Chem., Int. Ed., 2012, 51, 3147-3151.

18 S. Shen, J. Jiang, P. Guo, C. X. Kronawitter, S. S. Mao and L. Guo, Nano Energy, 2012, 1, 732-741.

19 R. Liu, Z. Zheng, J. Spurgeon and X. Yang, Energy Environ. Sci., 2014, 7, 2504-2517.

20 Y. Lin, S. Zhou, S. W. Sheehan and D. Wang, J. Am. Chem. Soc., 2011, 133, 2398-2401.

21 T. Hisatomi, H. Dotan, M. Stefik, K. Sivula, A. Rothschild, M. Graetzel and N. Mathews, Adv. Mater., 2012, 24, 2699-2702.

22 L. Steier, I. Herraiz-Cardona, S. Gimenez, F. FabregatSantiago, J. Bisquert, S. D. Tilley and M. Grätzel, Adv. Funct. Mater., 2014, 24, 7681-7688.

23 O. Moradlou, Z. Rabiei, A. Banazadeh, J. Warzywoda and M. Zirak, Appl. Catal., B, 2018, 227, 178-189.

24 J. Y. Kim, J.-W. Jang, D. H. Youn, J. Y. Kim, E. S. Kim and J. S. Lee, RSC Adv., 2012, 2, 9415-9422.

25 J. Deng, X. Lv, J. Gao, A. Pu, M. Li, X. Sun and J. Zhong, Energy Environ. Sci., 2013, 6, 1965-1970.

26 T. H. Wang, C.-C. Chiang, Y.-L. Wu, C. Lin, Y.-J. Cheng, Y.-K. Hsieh, C.-F. Wang and C. Huang, Appl. Catal., B, 2017, 207, 1-8.
27 M. P. Cardona, M. Li, W. Li, J. McCall, D. Wang, Y. Li and C. Yang, Mater.Today Energy, 2018, 8, 8-14.

28 A. Annamalai, A. G. Kannan, S. Y. Lee, D.-W. Kim, S. H. Choi and J. S. Jang, J. Phys. Chem. C, 2015, 119, 19996-20002.

29 Y. Liu, W. Li, J. Li, Y. Yang and Q. Chen, RSC Adv., 2014, 4, 3219-3225.

30 Y. Hou, Z. Wen, S. Cui, X. Feng and J. Chen, Nano Lett., 2016, 16, 2268-2277.

31 C. X. Guo, Y. Dong, H. B. Yang and C. M. Li, Adv. Energy Mater., 2013, 3, 997-1003.

32 Z. Liang, H. Hou, Z. Fang, F. Gao, L. Wang, D. Chen and W. Yang, ACS Appl. Mater. Interfaces, 2019, 11, 1916719175.

33 T.-Y. Yang, H.-Y. Kang, K. Jin, S. Park, J.-H. Lee, U. Sim, H.-Y. Jeong, Y.-C. Joo and K. T. Nam, J. Mater. Chem. A, 2014, 2, 2297-2305.

34 D. Wang, G. Chang, Y. Zhang, J. Chao, J. Yang, S. Su, L. Wang, C. Fan and L. Wang, Nanoscale, 2016, 8, 1269712701.

35 Z. Luo, T. Wang, J. Zhang, C. Li, H. Li and J. Gong, Angew. Chem., Int. Ed., 2017, 56, 12878-12882.

36 F. Le Formal, M. Grätzel and K. Sivula, Adv. Funct. Mater., 2010, 20, 1099-1107.

37 O. Zandi, J. A. Beardslee and T. Hamann, J. Phys. Chem. C, 2014, 118, 16494-16503.

38 Y. Wang and A. Hu, J. Mater. Chem. C, 2014, 2, 6921-6939.

39 D. Bhattacharya, M. K. Mishra and G. De, J. Phys. Chem. C, 2017, 121, 28106-28116.

40 V. Sharma, P. Tiwari and S. M. Mobin, J. Mater. Chem. B, 2017, 5, 8904-8924.

41 S. R. Pendlebury, A. J. Cowan, M. Barroso, K. Sivula, J. Ye, M. Grätzel, D. R. Klug, J. Tang and J. R. Durrant, Energy Environ. Sci., 2012, 5, 6304-6312.

42 K. Sivula, F. Le Formal and M. Grätzel, ChemSusChem, 2011, 4, 432-449.

43 P. Anilkumar, X. Wang, L. Cao, S. Sahu, J.-H. Liu, P. Wang, K. Korch, K. N. Tackett Ii, A. Parenzan and Y.-P. Sun, Nanoscale, 2011, 3, 2023-2027.

44 Y. Park, J. Yoo, B. Lim, W. Kwon and S.-W. Rhee, J. Mater. Chem. A, 2016, 4, 11582-11603.

45 L. Lin, X. Song, Y. Chen, M. Rong, T. Zhao, Y. Jiang, Y. Wang and X. Chen, Nanoscale, 2015, 7, 15427-15433.

46 R. Hu, L. Li and W. J. Jin, Carbon, 2017, 111, 133-141.

47 C. Hu, C. Yu, M. Li, X. Wang, Q. Dong, G. Wang and J. Qiu, Chem. Commun., 2015, 51, 3419-3422.

48 H. Luo, S. Dimitrov, M. Daboczi, J.-S. Kim, Q. Guo, Y. Fang, M.-A. Stoeckel, P. Samorì, O. Fenwick, A. B. Jorge Sobrido, X. C. Wang and M. Titirici, ACS Appl. Nano Mater., 2020, 3, 3371-3381.

49 C. Colliex, T. Manoubi and C. Ortiz, Phys. Rev. B: Condens. Matter Mater. Phys., 1991, 44, 11402.

50 C. Wang, Y. Wang, X. Liu, H. Yang, J. Sun, L. Yuan, G. Zhou and F. Rosei, Nanotechnology, 2015, 27, 035702.

51 Y. L. Chueh, M. W. Lai, J. Q. Liang, L. J. Chou and Z. L. Wang, Adv. Funct. Mater., 2006, 16, 2243-2251. 
52 D. K. Mann, J. Xu, N. E. Mordvinova, V. Yannello, Y. Ziouani, N. González-Ballesteros, J. P. Sousa, O. I. Lebedev, Y. V. Kolen'ko and M. Shatruk, Chem. Sci., 2019, 10, 2796-2804.

53 T. Liu, M. Morelli and Y. Li, Photoelectrochemical Solar Cells, 2018 pp. 159-218.

54 Y. Ling, G. Wang, J. Reddy, C. Wang, J. Z. Zhang and Y. Li, Angew. Chem., Int. Ed., 2012, 124, 4150-4155.

55 J. Wang, N. H. Perry, L. Guo, L. Vayssieres and H. L. Tuller, ACS Appl. Mater. Interfaces, 2019, 11, 2031-2041.

56 M. Omran, T. Fabritius, A. M. Elmahdy, N. A. Abdel-Khalek, M. El-Aref and A. E.-H. Elmanawi, Appl. Surf. Sci., 2015, 345, 127-140.

57 H. Zhang, Y. K. Kim, H. Y. Jeong and J. S. Lee, ACS Catal., 2018, 9, 1289-1297.

58 H. Zhang, W. Y. Noh, F. Li, J. H. Kim, H. Y. Jeong and J. S. Lee, Adv. Funct. Mater., 2019, 29, 1805737.

59 C. Tang, B. Sun, M. Li, J. Zhang, X. Fan, F. Gao, Y. Tong, L. Dong and Y. Li, J. Mater. Chem. A, 2019, 7, 8050-8054.

60 M. Li, Y. Yang, Y. Ling, W. Qiu, F. Wang, T. Liu, Y. Song, X. Liu, P. Fang and Y. Tong, Nano Lett., 2017, 17, 24902495.

61 Y. Ling, G. Wang, H. Wang, Y. Yang and Y. Li, ChemSusChem, 2014, 7, 848-853.

62 K. Sivula, R. Zboril, F. Le Formal, R. Robert, A. Weidenkaff, J. Tucek, J. Frydrych and M. Gratzel, J. Am. Chem. Soc., 2010, 132, 7436-7444.

63 R. Morrish, M. Rahman, J. D. MacElroy and C. A. Wolden, ChemSusChem, 2011, 4, 474-479.

64 Y. Ling, G. Wang, D. A. Wheeler, J. Z. Zhang and Y. Li, Nano Lett., 2011, 11, 2119-2125.
65 K. P. S. Parmar, H. J. Kang, A. Bist, P. Dua, J. S. Jang and J. S. Lee, ChemSusChem, 2012, 5, 1926-1934.

66 J. Y. Kim, H. Jun, S. J. Hong, H. G. Kim and J. S. Lee, Int. J. Hydrogen Energy, 2011, 36, 9462-9468.

67 F. Malara, A. Minguzzi, M. Marelli, S. Morandi, R. Psaro, V. Dal Santo and A. Naldoni, ACS Catal., 2015, 5, 52925300 .

68 J. Zhang, R. García-Rodríguez, P. Cameron and S. Eslava, Energy Environ. Sci., 2018, 11, 2972-2984.

69 B. Klahr, S. Gimenez, F. Fabregat-Santiago, T. Hamann and J. Bisquert, J. Am. Chem. Soc., 2012, 134, 4294-4302.

70 B. Klahr, S. Gimenez, F. Fabregat-Santiago, J. Bisquert and T. W. Hamann, Energy Environ. Sci., 2012, 5, 76267636.

71 B. Klahr and T. Hamann, J. Phys. Chem. C, 2014, 118, 10393-10399.

72 N. Yatom, O. Neufeld and M. Caspary Toroker, J. Phys. Chem. C, 2015, 119, 24789-24795.

73 K. Zhu, N. R. Neale, A. Miedaner and A. J. Frank, Nano Lett., 2007, 7, 69-74.

74 D. Monllor-Satoca, M. Bärtsch, C. Fàbrega, A. Genç, S. Reinhard, T. Andreu, J. Arbiol, M. Niederberger and J. R. Morante, Energy Environ. Sci., 2015, 8, 3242-3254.

75 Y. Tang and J. Arbiol, Nanoscale Horiz., 2019, 4, 12561276.

76 C. Ding, Z. Wang, J. Shi, T. Yao, A. Li, P. Yan, B. Huang and C. Li, ACS Appl. Mater. Interfaces, 2016, 8, 7086-7091.

77 L. M. Peter, L. H. Wong and F. F. Abdi, ACS Appl. Mater. Interfaces, 2017, 9, 41265-41272.

78 L. M. Peter, K. U. Wijayantha and A. A. Tahir, Faraday Discuss., 2012, 155, 309-322. 